Available online at GSC Online Press Directory

GSC Biological and Pharmaceutical Sciences

e-ISSN: 2581-3250, CODEN (USA): GBPSC2

Journal homepage: https://www.gsconlinepress.com/journals/gscbps

(RESEARCH ARTICLE)

\title{
Measuring IGF-1 and IGFBP-3 profiles in women seeking assisted reproduction; response of women categorized as poor-prognosis to recombinant growth hormone adjuvant therapy (Study 4)
}

\author{
John L Yovich ${ }^{1,2, *}$, Syeda Zaidi ${ }^{13}$, Minh DK Nguyen ${ }^{1}$, and Peter M Hinchliffe 1 \\ 1 PIVET Medical Centre Perth, Western Australia Australia 6007. \\ 2 Department of Pharmacy and Biomedical Sciences Curtin University Perth, Western Australia, Australia 6845. \\ ${ }^{3}$ Department of Obstetrics and Gynecology, Faculty of Medicine, University of Malaya Lembah Pantai, Kuala Lumpur \\ Malaysia 59100.
}

Publication history: Received on 15 October 2020; revised on 06 November 2020; accepted on 10 November 2020

Article DOI: https://doi.org/10.30574/gscbps.2020.13.3.0358

\begin{abstract}
In this fourth study examining the relevance of measuring the IGF profile (IGF-1, IGFBP-3 and the ratio of IGFBP-3/ IGF1) in women presenting for assisted reproductive technologies (ART), we have examined the influence of recombinant growth hormone $(\mathrm{rGH})$ prescribed as an adjuvant on the profile. Of 1633 ART-naïve women who completed an assessment cycle (AC) workup, and which included an IGF profile, 941 women proceeded to an in-vitro fertilization \pm intracytoplasmic sperm injection (IVF \pm ICSI) treatment cycle during the period January 2011 to December 2019. Among those women, 90 were prescribed rGH because they were classified as likely poor-prognosis cases on the basis of advanced age or evidence of severely reduced ovarian reserve parameters. These women had their IVF \pm ICSI treatment within 3-months of their AC and consented to a second IGF profile measurement 4-6 weeks during their rGH treatment phase. Of the 90 women prescribed rGH adjuvant, 71 used the adjuvant and 19 deferred the treatment, but still completed the second IGF-profile. The data showed that rGH caused a significant elevation in IGF-1 $(\mathrm{p}<0.0001)$ as well as its main binding protein, IGFBP-3 ( $\mathrm{p}<0.001)$, albeit to a lesser degree. Consequently, the IGF ratio, considered a more reliable marker of IGF-1 activity, was shown to be significantly reduced towards the normal range $(\mathrm{p}<0.0001)$. The data of clinical outcomes from these 90 women as well as the entire 941 women entering the IVF \pm ICSI treatment are presented for comparison, noting these data are not suitable for statistical evaluation as the groupings are disparate and unmatched. Further studies are intended to show the clinical relevance of increasing the IGF-1 levels in the analytical investigation of rGH as an adjuvant in ART.
\end{abstract}

Keywords: Human Growth Hormone (hGH); Insulin-Like Growth Factor-1 (IGF-1), Insulin-Like Growth Factor Binding Protein-3 (IGFBP-3); IGF Profile (IGF-1, IGFBP-3 and IGF Ratio); IGFBP-3/ IGF-1 Ratio (IGF Ratio); Assisted Reproductive Technology (ART); In Vitro Fertilization (IVF); Recombinant Growth Hormone (rGH); GH Adjuvant.

\section{Introduction}

Although assisted reproductive technology (ART) has contributed to an estimated more than 10 million women achieving live births worldwide over the past 42 years, the successes have occurred mainly in young women, leaving an unmet need among those women over 35 years. Such women form an increasing proportion of those classified as poorprognosis cases, yet they have high expectation and are prepared to invest time, finances and emotional energy into the quest for a successful pregnancy. The Annual Capri Workshop Group, which comprises many of the long-standing

${ }^{*}$ Corresponding author: John L Yovich

PIVET Medical Centre Perth, Western Australia Australia 6007.

Copyright (@ 2020 Author(s) retain the copyright of this article. This article is published under the terms of the Creative Commons Attribution Liscense 4.0. 
workers in ART from Europe along with a few from The United Kingdom and the United States of America, recently reported on the top 10 uncertainties remaining and where quality research is required [1]. The Group have been highly critical of the over-use of ART where the prognosis is poor, particularly for women classified as poor ovarian responders, and are especially critical of the use of add-ons and adjuvants where the scientific data is not clearly supportive, meaning the presented data fails to meet the highest level of evidence-based medical standards. Over the past 10 years our PIVET group, a long-established clinical and research facility, has been reporting on the use of one of these adjuvants, namely recombinant growth hormone ( $\mathrm{rGH})[2,3,4]$. We have been exploring the notion that one of the underlying causes of poor-prognosis may be attributed to adult growth hormone deficiency (AGHD) [2,3,4]. A recent evaluation of the data from 42 published studies utilizing rGH between the years 2000-2019, indicates they were all undertaken on an empirical basis [5,6] without sound scientific foundation, albeit 50\% of studies reported clinical benefits. However, a few of the $\mathrm{rGH}$ articles did report some relevant beneficial effects in laboratory studies, one implying a direct action in improving oocyte quality, acting via upregulation of the GH receptor (GHR) in the oocyte as well as the demonstration of improved mitochondrial function within the aging oocytes [7]. A more recent laboratory study confirms the upregulation of, not only the GHR in the granulosa cells of older women, but also the gonadotrophin receptors, follicle stimulating hormone receptor (FSHR) and luteinizing hormone receptor (LHR), as well as bone morphogenetic hormone receptor (BMPR). These upregulations, with associated modulations in their profiles of expression, were associated with improved clinical outcomes for those women [8]. However, in order to apply a more definitive solution to the notion of AGHD as a cause of poor-prognosis we have been sequentially exploring the relevance of both human growth hormone (hGH) estimations in serum along with that of the insulin growth factor (IGF) profile; specifically embracing IGF-1, its main binding protein IGFBP-3 and the IGF Ratio of IGFBP-3/IGF [9]. These studies follow on the clinical standards for the diagnosis of growth hormone deficiency (GHD) in children [10] along with the recent acceptance by the Endocrine Society that AGHD is a legitimate endocrine disorder, distinct from the natural aging process [11].

The studies we have so far reported include an evaluation of the IGF profile against clinical parameters [12] as well as against the accepted parameters of ovarian reserve, namely the antral follicle count (AFC) and the serum measurement of anti-Mullerian hormone (AMH) [13]. These have been shown to have prognostic relevance in various outcomes from in-vitro fertilization (IVF), particularly ovarian responsiveness, oocyte numbers and oocyte quality. Most recently we have also reported on the relevance of hGH testing on the same serum samples used for the IGF profile, noting that only $12 \%$ of measurements fall into the AGHD-exclusion zone [9]. Furthermore, whilst both the IGF profile and the hGH levels have a significant association with the woman's age, hGH levels appear to be disconnected from other clinical parameters such as stature and body mass index (BMI) as well as the ovarian reserve parameters, AFC and AMH [13]. This next study is designed to test the hypothesis that $\mathrm{rGH}$, given as an adjuvant for IVF treatments, can influence the IGF profile.

\section{Material and methods}

As previously reported, clinical protocols for ART treatments at PIVET are conducted according to well described protocols and the application of clinical algorithms $[14,15,16]$. The optimum workup of IVF-naïve women presenting for ART requires an Assessment Cycle (AC) during which a profile of tests are undertaken for the evaluation of ovulation, $\mathrm{AFC}$ and $\mathrm{AMH}$ for the ovarian reserve, pelvic anatomical features by trans-vaginal ultrasound, periovulatory cervical mucus features, a post-coital evaluation and mid-luteal hormonal measurements. Our preferred evaluation of tubal patency is by hystero-salpingo-contrast sonography (HyCoSy), but hysterosalpingography can also suffice. Recently we have included IGF profiling on day $5 \pm 1$ of the follicular phase. Figure 1 covers the study period from January 2011 to December 2019 and shows the derivation of ACs where IGF profiles have also been completed ( $n=1633$ cycles). Of these women 941 proceeded into an IVF \pm ICSI treatment cycle, 98 of whom utilized rGH adjuvant, mainly for advanced female age ( $>40$ years) or because very low AFC counts and AMH levels predicted a poor prognosis. For this particular study (study 4) we have asked those women who had been offered rGH as an adjuvant to have a further IGF profile performed 4-6 weeks after commencing the daily rGH injections; i.e. whilst on the adjuvant hormone injections. Ninety women consented and were prescribed rGH adjuvant, but 19 of these women subsequently decided to defer utilizing rGH although they agreed to complete the second IGF profile, which all 90 women undertook. 


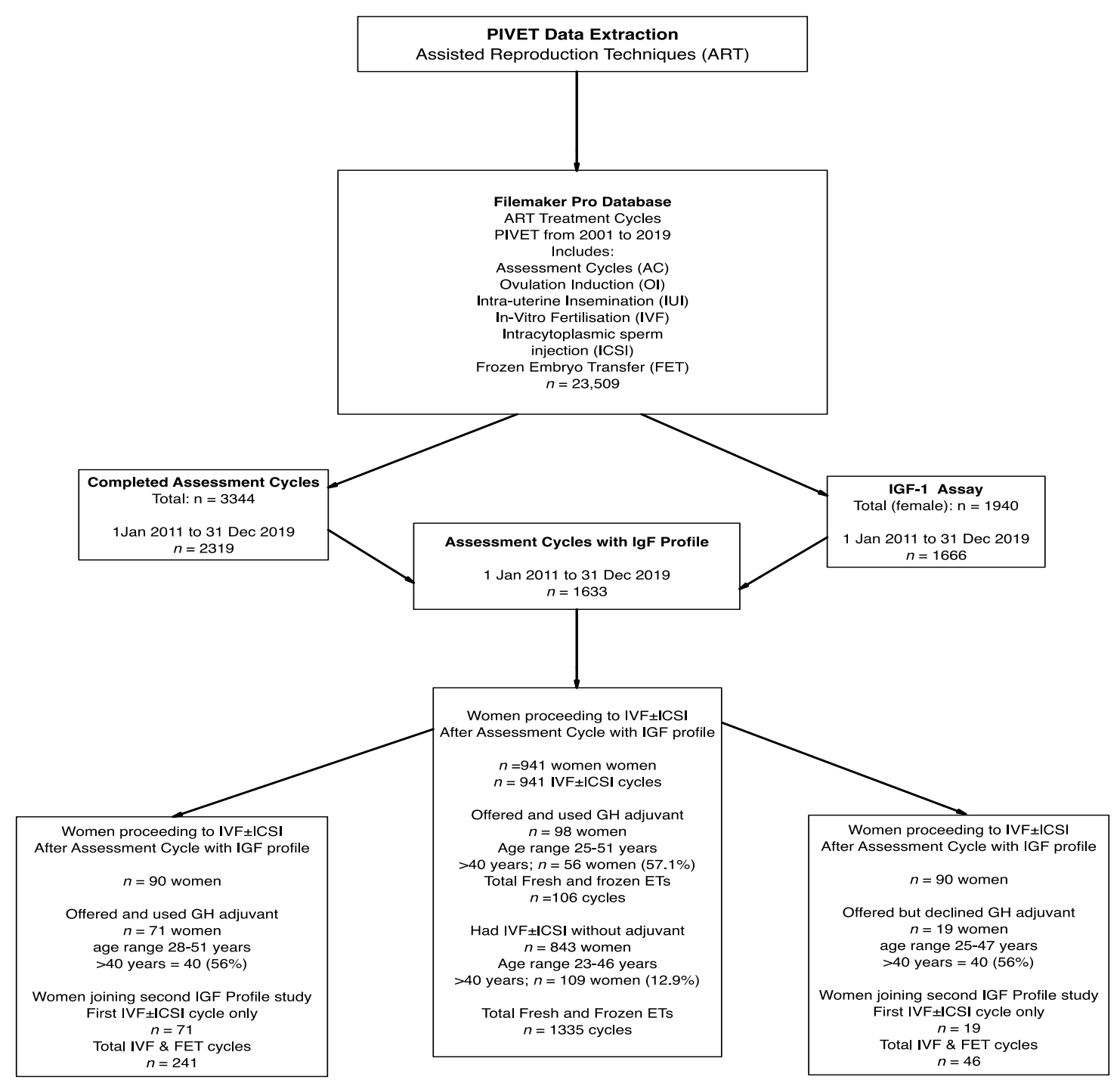

Figure 1 Flowchart showing the derivation of the 90 women who had a second IGF profile after prescription of GH adjuvant therapy.

\subsection{PIVET FSH-dosing algorithm}

Following the AC, couples are reviewed to plan their specific fertility treatment which may include preliminary operative treatments for females, including hysteroscopy for uterine polyps and laparoscopy for myomectomy, treatment of endometriosis and adenomyosis if the uterus is very bulky. Thereafter, those proceeding to IVF have ovarian stimulation protocols which base the FSH-dosage on various PIVET algorithms. These algorithms have been reported and validated from various studies since 2010, covering the entire period of the current study $[14,15,16]$. One such Algorithm is depicted in Table 1 and has been trialed at PIVET [17]. The algorithms rely mainly on the woman's age and her AFC category, with adjustments according to her serum AMH grouping and modified from an array of 6 other factors, including her BMI, smoking history, base-line (Day-2) FSH level and decisions such as luteal phase support and oocyte or embryo cryopreservation plans. These algorithms ensure the generation of $10 \pm 2$ oocytes for $>80 \%$ of cycles, dosages rarely need changing and ovarian hyperstimulation syndrome occurs rarely, currently $0.1 \%$ of those IVF cases proceeding to oocyte pick-up (OPU). 
Table 1 One of the PIVET FSH-dosing Algorithms which have been applied throughout this study period; this one being particularly suited to the Bemfola ${ }^{\circledR}$ pen (Gedeon Richter; Sydney, NSW, Australia) which provides 12.5 IU increments of rFSH, shown to be biosimilar to Gonal-f® (follitropin alpha; Merck, Sydney, NSW, Australia) and has been validated at PIVET [17].

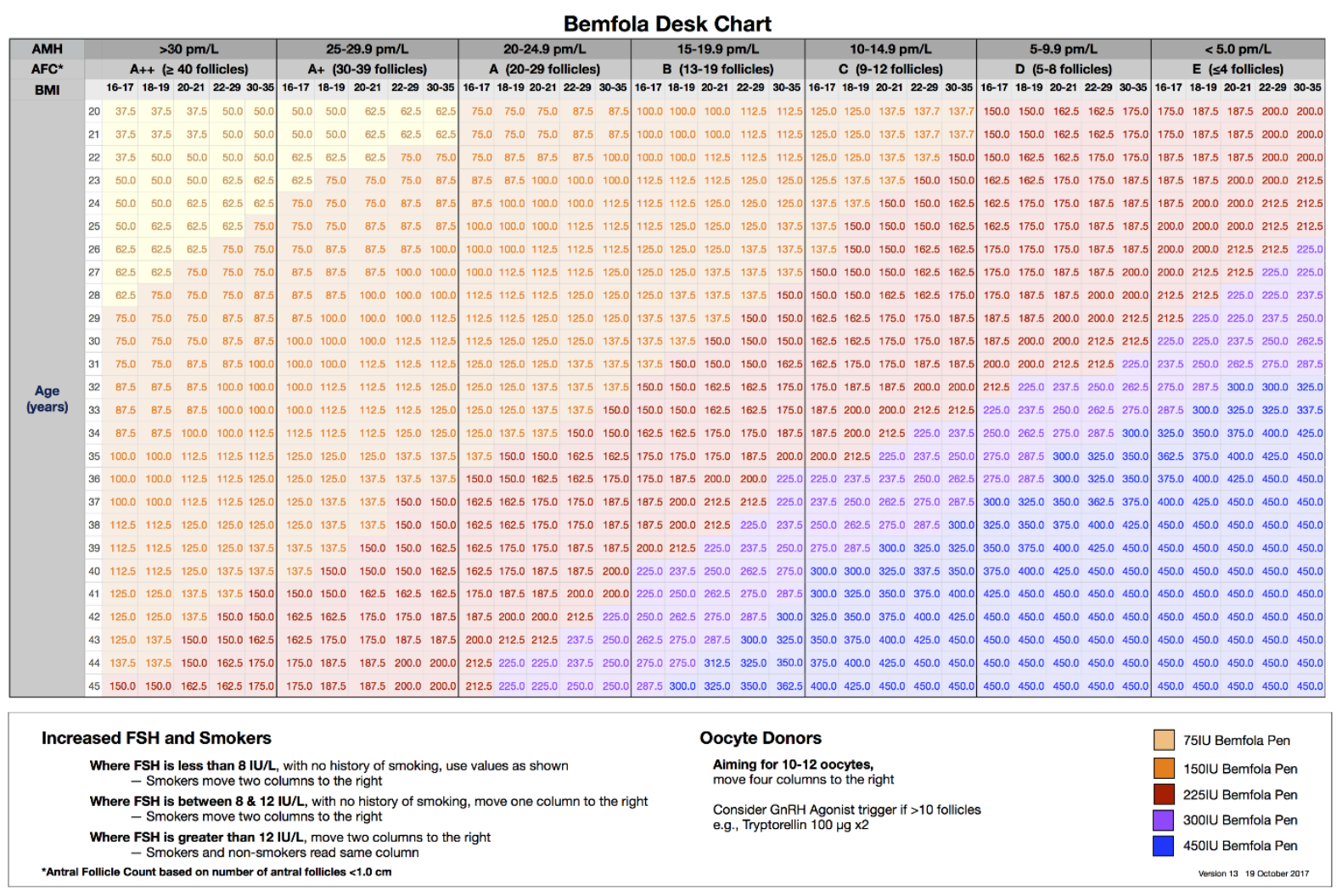

\subsection{Ovarian Reserve Testing}

The AFC assessment is undertaken around Day- 5 of the AC by trans-vaginal ultrasound, initially using the Voluson 730 Expert for 5 years followed by the Voluson P6 in the recent 4-year period. (Both machines were supplied by General Electric, Australia). The enzyme-based AMH assays commenced with the Beckman Coulter Immunotech Gen 2 Elisa platform (Danaher Corporation; California, USA) and subsequently using the Cobas Elecsys e411 platform (Roche Diagnostics, Basel, Switzerland). These assays have been fully described in Study 2 [13] and have been shown to be tightly correlated. The laboratories at PIVET are accredited with the National Australian Testing Authority (NATA) following annual assessment which includes evidence of participation in approved quality assurance programs.

\section{3. rGH adjuvant protocol}

As previously reported, $\mathrm{rGH}$ is considered for those women classified as poor-prognosis from suboptimal outcomes, including poor ovarian responses [5] in previous IVF treatments, or considered to be highly likely to have a poor prognosis on the basis of advanced age ( $>40$ years), or low ovarian reserve (being in the E category of the PIVET FSHdosing algorithm). In this study the indications for adjuvant $\mathrm{rGH}$ was advanced maternal age ( $>40$ years) and low AMH and AFC category (Group E). Those cases prescribed rGH are currently treated with the SciTropin A pen (SciGen, Sydney, Australia) delivering 1 IU recombinant somatotropin (rGH) subcutaneous each day (by 6-clicks of the pen) commencing in the early days of the menstrual cycle preceding the OPU procedure. This is continued until the Day of Trigger, usually Day-12 of the OPU cycle, although women are advised to complete their pen even into the days of OPU and luteal phase to avoid wastage. The Scitropin cartridge supplies 15 days of $\mathrm{rGH}$ and costs around \$A300. Most patients will use 3 pens in the lead-up to their OPU. Those who agree to the extra blood tests for a repeat IGF profile tend to do so near the time of Trigger, i.e. 4-6 weeks into the rGH treatment and 2-4 months after the first IGF profile performed during the AC.

\subsection{PIVET IVF program}

PIVET is one of the pioneer units having established in 1980, generating successful pregnancies in 1981 in Western Australia [18,19]. Corresponding author JLY had already established an IVF facility in London, generating an early pregnancy in 1979. In keeping with the Code of Practice put forward by the Reproductive Technology Accreditation 
Committee (RTAC) under the auspices of the Fertility Society of Australia (FSA), PIVET is committed to a single embryo transfer (SET) protocol (95\%), blastocyst culture and transfer (85\%) and cryopreservation of blastocysts using the Cryotop vitrification process (supplied by Kitazato, Tokyo, Japan) [20]. The 11-year study period (2011-2019) is recognized as a highly stable and effective period at PIVET where annual Australia and New Zealand Assisted Reproduction Database (ANZARD) reports indicate PIVET ART-outcomes are consistently in the top quartile for livebirths arising from ART treatment cycles initiated [21].

\subsection{Pregnancies}

At PIVET all ART cycles are monitored through the luteal phase to Day 19 after the hCG Trigger (for IVF cycles) or after the commencement of progesterone pessaries in frozen embryo transfer (FET) cycles which are predominantly managed under a hormone replacement schedule (HRT) for logistic benefits. An hCG Trigger is also used for non-HRT FET cycles managed by natural cycle tracking or following low-dose FSH stimulation. Once pregnancy is diagnosed women have a blood hormonal profile checked each week for an E2, P4 and B-hCG profile and undertaking a transvaginal pelvic scan at 7-weeks to determine an intra-uterine gestational sac, expectantly containing a live fetus. PIVET has an active hormonal support protocol for early pregnancy management [16] and, although women have their further management after the first trimester by external consultant obstetricians, all women are tracked by PIVET through to the final pregnancy outcome. To gain a proper perspective on treatment outcomes, PIVET applies a productivity index, being the cumulative pregnancies and live births arising from a single oocyte retrieval cycle, taking into account every embryo transfer (both fresh and subsequent frozen embryos) resulting from the one initiated IVF cycle.

\subsection{Statistics}

Data extractions from the Filemaker database were placed in Microsoft Excel spreadsheets and sorted according to the relevant tests. Thereafter the sorted data was placed in the application Past 4.03 (developed by Øyvind Hammer) [22] for statistical data analysis. This application also generated the Tables comprising the statistical summaries, finally placed in Microsoft Word for clearer display. Skewness, as a measure of divergence from the Normal, Gaussian profile is considered to be acceptable (non-divergent) in the range of -1 to +1 . This follows from applying the Fisher-Pearson coefficient which analyses the number of points outside the standard deviation. Kurtosis is a measure of whether the spread of data (about the mean) are heavy-tailed or light-tailed relative to a classical bell-shaped curve. The acceptable range for a classical normal distribution accepts kurtosis under \pm 3.0 , preferably under -2 to +2 in univariate studies. Having demonstrated that the data comprising the IGF profile (IGF-1, IGFBP-3 and IGF Ratio) are all distributed in a Normal, or near Normal fashion, the relationship among the means was examined by Student's t-test for 2 data-sets, but also by one-way ANOVA for overall comparison with >data-2 sets. Both Mann-Whitney and Tukey's pair-wise plots compared the individual means in those data which displayed elevated kurtosis above \pm 2.0 . The Kruskal-Wallis test was applied to examine equality between sample medians and Mann-Whitney applied for pairwise comparisons between individual sub-groups. Bonferroni correction was applied for sub-group comparisons. Chi-squared tests were applied for $2 \mathrm{x} 2$ comparisons within contingency tables. Following corrections, probability values of $\mathrm{p}<0.05$ were considered significant for any test. The Past 4.03 application also generated the Figures which were then upgraded in the xDiagram 5.4 application (developed by Vu Tien Thinh) enabling optimal display for this publication.

\section{Results}

The IGF profile among 1633 women who completed an AC profile, showed that the data for all 3 components, namely IGF-1, IGFBP-3 and IGF ratio, were distributed in a Normal, Gaussian manner, enabling parametric analysis of the data [12]. The data presented in this study for the IGF profile are drawn from that same group of women as a sub-set of 90 cases who were prescribed rGH as potential poor-prognosis cases.

\subsection{IGF-1 levels}

The IGF-1 levels undertaken 4-6 weeks after commencing Scitropin A rGH as an adjuvant in IVF treatment is shown in Table 2. The levels for 71 women are compared with those measured during the preliminary AC and are compared with similar measurements undertaken in 19 women who were offered rGH adjuvant but decided to defer using it pending the outcomes of this IVF treatment cycle. It can be seen that the mean levels of IGF-1 rose from $24.7 \mathrm{nmol} / \mathrm{L}$ to 34.7 $\mathrm{nmol} / \mathrm{L}$ under $\mathrm{rGH}$ influence, whereas the mean levels remained at $26.7 \mathrm{nmol} / \mathrm{L}$ (from $26.8 \mathrm{nmol} / \mathrm{L}$ ) in those women who did not use the prescribed rGH adjuvant. Similarly, the median levels rose from $24 \mathrm{nmol} / \mathrm{L}$ to $34 \mathrm{nmol} / \mathrm{L}$ under the influence of $\mathrm{rGH}$ adjuvant whilst the median levels were unchanged at $27 \mathrm{nmol} / \mathrm{L}$ for both measurements in those women who did not use the rGH adjuvant. The fact that mean and median levels were similar, and both skewness and kurtosis were very low $(<1.0 \mathrm{nmol} / \mathrm{L})$ is consistent with our previous Study 1 report from women undertaking ACs at 
PIVET, that these data are distributed in a Normal, Gaussian fashion [12]. Applying Student's t-test indicates the elevation of IGF-1 under rGH influence, is a highly significant observation $(\mathrm{p}<0.0001)$ whereas there was no change among the 19 women who declined to use their prescribed rGH adjuvant. These effects are depicted in Figure 2.

Table 2 Summary statistics for the 90 women who had 2 measurements of the IGF profile taken before and during the rGH adjuvant therapy period in the 6-week lead-up to an IVF \pm ICSI treatment cycle. This table shows the statistical profile for IGF-1 levels among those women who did inject rGH daily $(n=71)$ and those who declined ( $n=19)$ but still undertook the second IGF profile measurement.

\begin{tabular}{|l|l|l|l|l|}
\hline \multicolumn{6}{|l}{ IGF-1 Levels nmol/L vs Growth Hormone Treatment Status } \\
\hline GH status & IGF-1 GH- & IGF-1 GH+ & IGF-1 GH- & IGF-1 GH- \\
\hline Women tested & 71 & 71 & 19 & 19 \\
\hline Min & 12 & 21 & 14 & 13 \\
\hline Max & 41 & 49 & 39 & 37 \\
\hline Sum & 1752 & 2464 & 509 & 507 \\
\hline Mean & 24.7 & 34.7 & 26.8 & 26.7 \\
\hline Std. error & 0.74 & 0.83 & 1.69 & 1.42 \\
\hline Variance & 39.3 & 48.8 & 54.5 & 38.6 \\
\hline Stand. dev & 6.27 & 6.98 & 7.38 & 6.21 \\
\hline Median & 24 & 34 & 27 & 27 \\
\hline 25th centile & 21 & 30 & 23 & 22 \\
\hline 75th centile & 29 & 39 & 32 & 32 \\
\hline Skewness & 0.27 & 0.27 & -0.24 & -0.36 \\
\hline Kurtosis & -0.28 & -0.38 & -0.58 & -0.13 \\
\hline Geom. mean & 23.87 & 34.01 & 25.71 & 25.91 \\
\hline Coeff. var & 25.39 & 20.13 & 27.56 & 23.27 \\
\hline Statistics & $\mathrm{p}<0.0001$ & & $\mathrm{p}=$ n.s. & \\
\hline
\end{tabular}

\subsection{IGFBP-3 levels}

The IGFBP-3 levels undertaken 4-6 weeks after commencing Scitropin A rGH as an adjuvant in IVF treatment is shown in Table 3. The levels for 71 women are compared with those measured during the preliminary AC and are compared with similar measurements undertaken in 19 women who were offered $\mathrm{rGH}$ adjuvant but decided to defer using it pending the outcomes of this IVF treatment cycle. It can be seen that the mean levels of IGF-1 rose from $171.1 \mathrm{nmol} / \mathrm{L}$ to $189.0 \mathrm{nmol} / \mathrm{L}$ under $\mathrm{rGH}$ influence, whereas the mean levels showed minimal change at $168.6 \mathrm{nmol} / \mathrm{L}$ (from 171.1 $\mathrm{nmol} / \mathrm{L}$ ) in those women who did not use the prescribed rGH adjuvant. Similarly, the median levels rose from 171 $\mathrm{nmol} / \mathrm{L}$ to $183 \mathrm{nmol} / \mathrm{L}$ under the influence of rGH adjuvant whilst the median levels were unchanged at $188 \mathrm{nmol} / \mathrm{L}$ to $187 \mathrm{nmol} / \mathrm{L}$ in those women who did not use the rGH adjuvant. The fact that mean and median levels were similar, and both skewness and kurtosis were very low ( $<1.0 \mathrm{nmol} / \mathrm{L})$ is, like the IGF-1 levels, consistent with our previous Study 1 report from women undertaking ACs at PIVET, that these data are distributed in a Normal, Gaussian fashion [12]. Again, applying Student's t-test indicates the elevation of IGFBP-3 under rGH influence, is a highly significant observation $(p<0.001)$ whereas there was no change among the 19 women who declined to use their prescribed rGH adjuvant. These effects are depicted in Figure 3. 


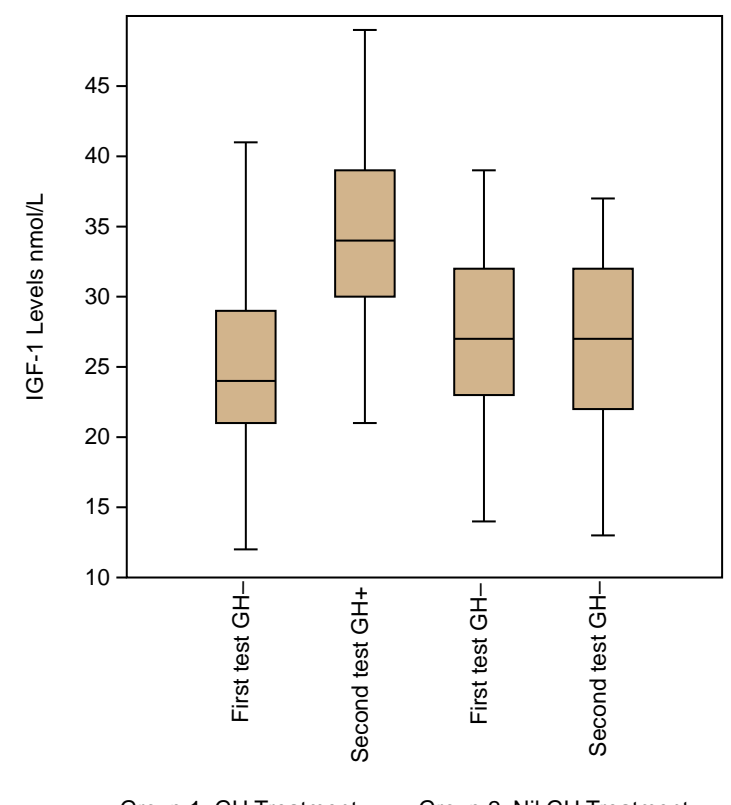

Figure 2 Box-plot display of the IGF-1 levels for the 90 women who completed two IGF profiles; prior and during prescribed $\mathrm{rGH}$ adjuvant therapy in the lead-up to an IVF \pm ICSI treatment cycle. The data is derived from Table 2.

Table 3 Summary statistics for the 90 women who had 2 measurements of the IGF profile taken before and during the rGH adjuvant therapy period in the 6-week lead-up to an IVF \pm ICSI treatment cycle. This table shows the statistical profile for IGFBP-3 levels among those women who did inject rGH daily $(n=71)$ and those who declined ( $n=19)$ but still undertook the second IGF profile measurement.

\begin{tabular}{|l|l|l|l|l|}
\hline \multicolumn{6}{|l}{ IGFBP-3 Levels nmol/L vs Growth Hormone Treatment Status } \\
\hline GH Status & IGFBP-3 GH- & IGFBP-3 GH+ & IGFBP-3 GH- & IGFBP-3 GH- \\
\hline Women tested & 71 & 71 & 19 & 19 \\
\hline Min & 118 & 125 & 128 & 123 \\
\hline Max & 253 & 271 & 226 & 233 \\
\hline Sum & 12149 & 13420 & 3250 & 3204 \\
\hline Mean & 171.1 & 189.0 & 171.1 & 168.6 \\
\hline Std. error & 3.55 & 3.86 & 5.45 & 6.55 \\
\hline Variance & 893.5 & 1058.0 & 565.1 & 814.5 \\
\hline Stand. dev & 29.89 & 32.53 & 23.77 & 28.54 \\
\hline Median & 171 & 183 & 172 & 171 \\
\hline $25^{\text {th }}$ centile & 150 & 167 & 149 & 140 \\
\hline $75^{\text {th }}$ centile & 187 & 215 & 188 & 187 \\
\hline Skewness & 0.53 & 0.31 & 0.19 & 0.21 \\
\hline Kurtosis & -0.03 & -0.63 & 0.30 & -0.06 \\
\hline Geom. mean & 168.61 & 186.28 & 169.48 & 166.33 \\
\hline Coeff. var & 17.47 & 17.21 & 13.90 & 16.92 \\
\hline Statistics & $p<0.001$ & & $p=n . s$. & \\
\hline
\end{tabular}




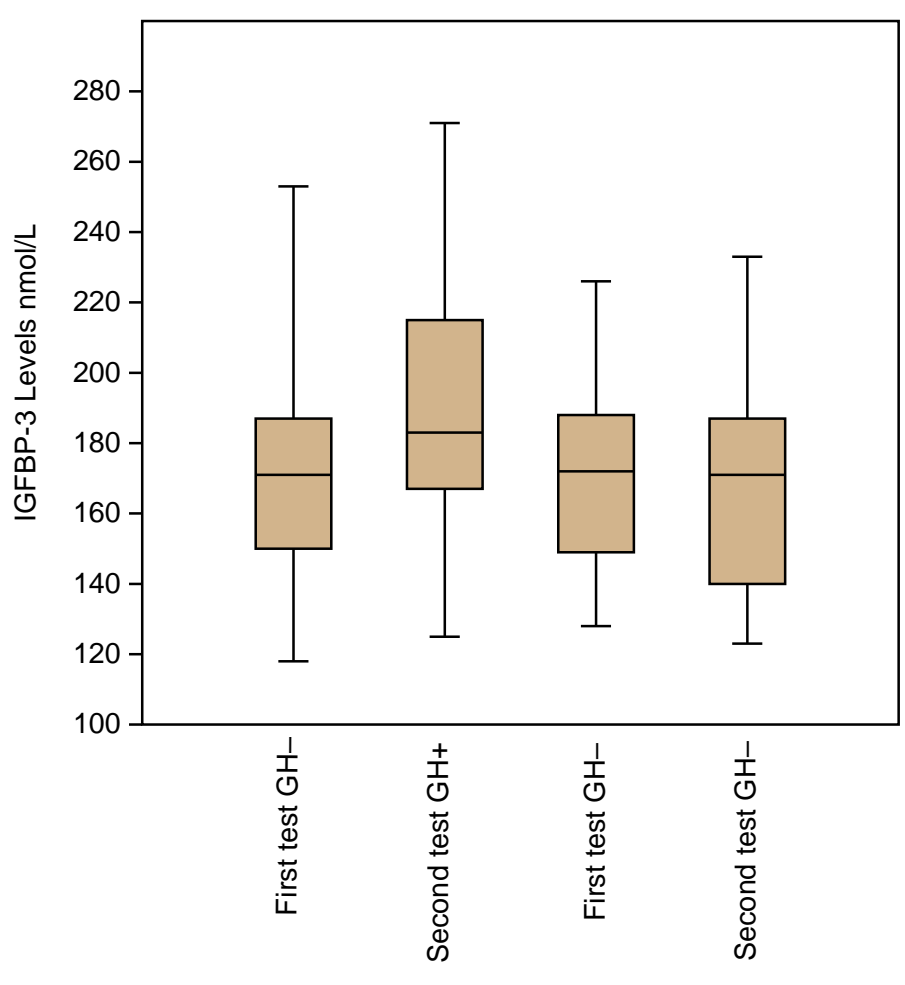

Group 1: GH Treatment

Group 2: Nil GH Treatment

Figure 3 Box-plot display of the IGFBP-3 levels for the 90 women who completed two IGF profiles; prior and during prescribed rGH adjuvant therapy in the lead-up to an IVF \pm ICSI treatment cycle. The data is derived from Table 3.

\subsection{IGF ratio}

Our calculation of the IGF ratio utilizes the formula IGFBP-3/ IGF-1. The IGF ratios undertaken 4-6 weeks after commencing Scitropin A rGH as an adjuvant in IVF treatment is shown in Table 4. The levels for 71 women are compared with those measured during the preliminary AC and are compared with similar measurements undertaken in 19 women who were offered rGH adjuvant but decided to defer using it pending the outcomes of this IVF treatment cycle. It can be seen that the mean IGF ratio declined from 7.3 to 5.6 under rGH influence, whereas the mean IGF ratio showed minimal change at 6.6 (from 6.7) in those women who did not use the prescribed rGH adjuvant. Similarly, the median levels declined from 6.8 to 5.4 under the influence of rGH adjuvant whilst the median levels were essentially unchanged at 6.7 (from 6.4) in those women who did not use the rGH adjuvant. The fact that mean and median levels were similar, and both skewness and kurtosis were very low $(<1.0 \mathrm{nmol} / \mathrm{L})$ is, like the IGF-1 levels and IGFBP-3 levels, consistent with our previous Study 1 report from women undertaking ACs at PIVET, that these data are distributed in a Normal, Gaussian fashion [12]. Again, applying Student's t-test indicates the lowering of the IGF ratio (towards the normal range) under $\mathrm{rGH}$ influence, is a highly significant observation $(\mathrm{p}<0.001)$ whereas there was no change among the 19 women who declined to use their prescribed rGH adjuvant. These effects are depicted in Figure 4. 


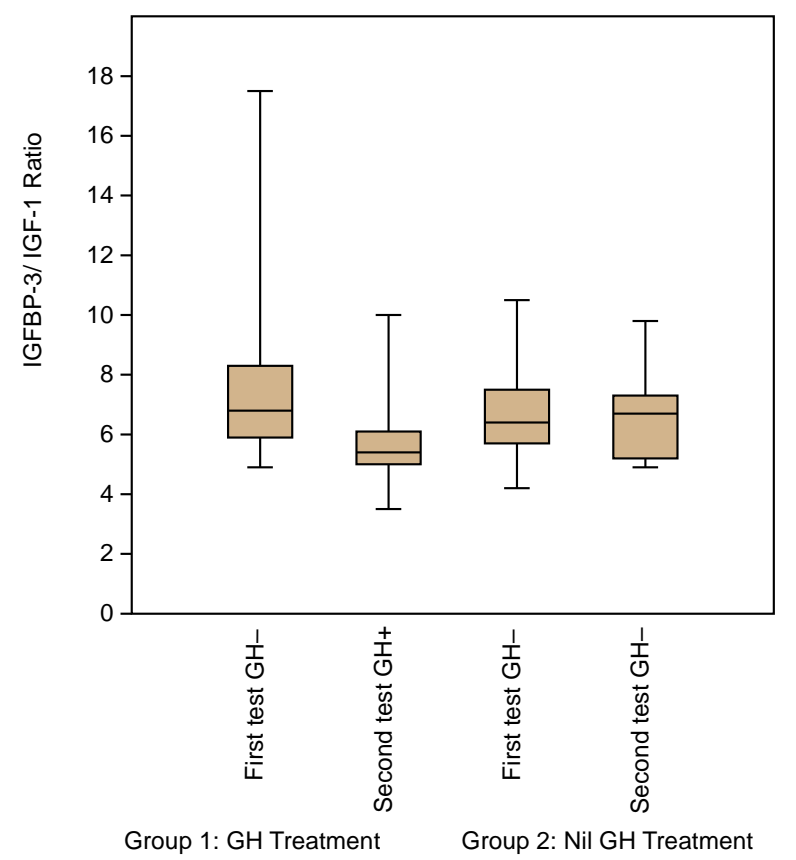

Table 4 Summary statistics for the 90 women who had 2 measurements of the IGF profile taken before and during the rGH adjuvant therapy period in the 6-week lead-up to an IVF \pm ICSI treatment cycle. This table shows the statistical profile for the IGF ratio (IGFBP-3/IGF-1) among those women who did inject rGH daily $(n=71)$ and those who declined $(\mathrm{n}=19)$ but still undertook the second IGF profile measurement.

\begin{tabular}{|l|l|l|l|l|}
\hline \multicolumn{6}{|l}{ IGFBP-3/ IGF-1 Ratio vs Growth Hormone Treatment Status } \\
\hline GH Status & IGF Ratio GH- & IGF Ratio GH+ & IGF Ratio GH- & IGF Ratio GH- \\
\hline Women tested & 71 & 71 & 19 & 19 \\
\hline Min & 4.9 & 3.5 & 4.2 & 4.9 \\
\hline Max & 17.5 & 10 & 10.5 & 9.8 \\
\hline Sum & 518.7 & 395.6 & 127.9 & 124.4 \\
\hline Mean & 7.3 & 5.6 & 6.7 & 6.6 \\
\hline Std. error & 0.24 & 0.12 & 0.35 & 0.31 \\
\hline Variance & 4.1 & 1.1 & 2.3 & 1.8 \\
\hline Stand. dev & 2.04 & 1.04 & 1.50 & 1.35 \\
\hline Median & 6.8 & 5.4 & 6.4 & 6.7 \\
\hline $25^{\text {th }}$ centile & 5.9 & 5 & 5.7 & 5.2 \\
\hline $75^{\text {th }}$ centile & 8.3 & 6.1 & 7.5 & 7.3 \\
\hline Skewness & 2.18 & 1.25 & 0.80 & 0.66 \\
\hline Kurtosis & 8.05 & 3.81 & 0.82 & 0.32 \\
\hline Geom. mean & 7.08 & 5.48 & 6.58 & 6.42 \\
\hline Coeff. var & 27.87 & 18.71 & 22.35 & 20.68 \\
\hline Statistics & $p<0.0001$ & & $p=n . s$. & \\
\hline
\end{tabular}




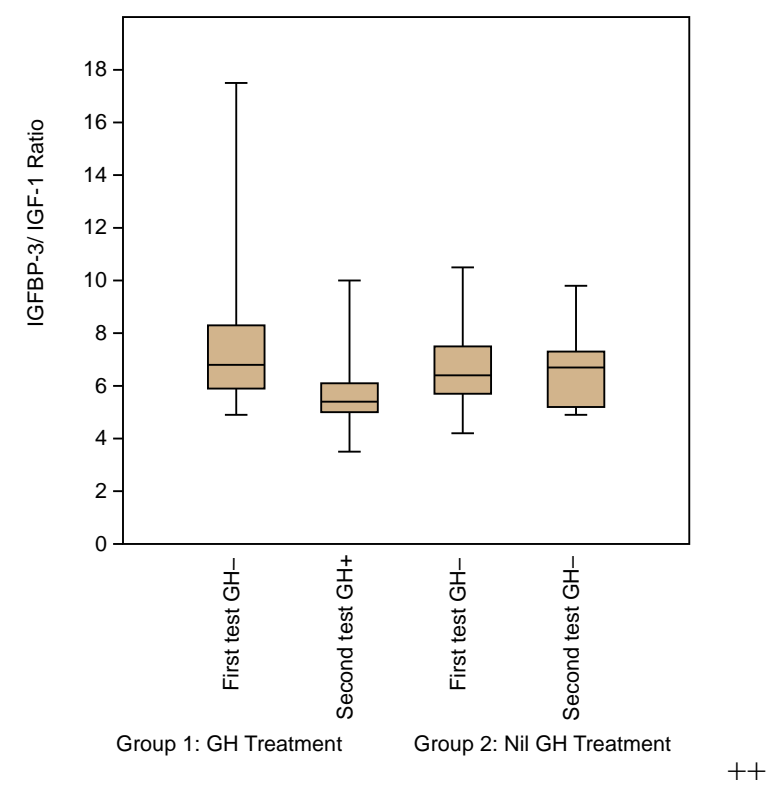

Figure 4 Box-plot display of the IGF ratio for the 90 women who completed two IGF profiles; prior and during prescribed $\mathrm{rGH}$ adjuvant therapy in the lead-up to an IVF \pm ICSI treatment cycle. The data is derived from Table 4.

\subsection{IVF Treatment outcomes}

It has been calculated that meaningful studies involving randomised controls require a minimal 400 cases divided randomly between those who did, or did not, receive the adjuvant [23]. Although the case numbers in each of the two groups is not sufficient to draw significant conclusions regarding any benefits between utilising the rGH adjuvant or declining to use it; a few observations are presented here for completeness.

Table 5 Summary statistics for the 90 women who had 2 measurements of the IGF profile taken before and during the rGH adjuvant therapy period in the 6-week lead-up to an IVF \pm ICSI treatment cycle. This table shows the statistical profile for the treatment cycle outcomes among those women who did inject rGH daily $(n=71)$ and those who declined $(n=19)$ but still undertook the second IGF profile measurement.

\begin{tabular}{|l|l|l|}
\hline & $\mathbf{7 1} \mathbf{G H +}$ & $\mathbf{1 9} \mathbf{G H}-$ \\
\hline ART cycles (IVF \& FETs; $n$ ) & 241 & 46 \\
\hline ART cycles/ woman & 3.4 & 2.4 \\
\hline Age range at cycle (years) & $28-51$ & $25-47$ \\
\hline Oocyte/OPU ( $n$ ) & 7.4 & 9.3 \\
\hline Oocyte utilisation rate & $22.4 \%$ & $21.6 \%$ \\
\hline Embryo utilisation rate & $44.7 \%$ & 39.4 \\
\hline Pregnancy productivity rate/ Initiated cycle & $24.1 \%$ & $19.6 \%$ \\
\hline Pregnancy productivity rate / OPU & $26.9 \%$ & $22.0 \%$ \\
\hline Pregnancy productivity rate / ET & $37.7 \%$ & $42.9 \%$ \\
\hline Miscarriage \pm ectopic rate & $8.3 \%$ & $4.3 \%$ \\
\hline Livebirth productivity rate / Initiated cycle & $12.0 \%$ & $15.2 \%$ \\
\hline Livebirth productivity rate / OPU & $13.4 \%$ & $17.1 \%$ \\
\hline Livebirth productivity rate / ET & $18.8 \%$ & $23.3 \%$ \\
\hline
\end{tabular}

IVF: invitro fertilization; FET: frozen embryo transfer; OPU: oocyte pick-up; ET: embryo transfer 
The treatment outcomes on the 90 fresh IVF cycles $( \pm \mathrm{rGH})$ revealed 14 pregnancies in the rGH treated group ( $\mathrm{n}=71$ women) with 8 proceeding to live births, one birth delivering healthy twins. However, 5 of the treatment cycles resulted in freeze-all embryos as per PIVET policy when $\geq 15$ oocytes are recovered. In the smaller group of women who did not take the rGH adjuvant which was prescribed ( $n=19$ women), there were 3 pregnancies, but only one proceeded to a live birth. There were 2 treatment cycles with freeze-all embryos. The full profile of outcomes is summarized in Table 5. It can be seen that the oocyte utilization rate was similar in both groups, with embryo utilization apparently higher among the women who received the rGH adjuvant. With respect to pregnancy and live births, the data set was too small to subanalyze with respect to the most important variable that of the women's age groupings. However, in this small data set we believe the productivity rates per initiated cycle provide the most relevant figure, actually being similar for both groups.

After a difficult start in the 1970's characterized by a poor-prognosis for the majority (>90\%) of women attempting to resolve their infertility by undertaking IVF treatment [18,19], the wider field of ART embracing cryopreservation, ICSI and tighter laboratory management has seen the prognosis improve dramatically. Reference to Table 6, second column, shows that $>50 \%$ of women across ages $25-47$ years initiating treatment can expect a live baby from a single IVF cycle involving OPU. On the other hand, the first column in Table 6 shows that $10 \%$ of women can be seen to have a poorer prognosis where the live birth rate is $21 \%$ of those reaching OPU. In past studies, such cases have been defined by poor ovarian responses (POR) and repetitive implantation failures (RIF) in previous IVF cycles [5].

Table 6 Summary statistics of the clinical outcomes for the 941 women who had completed an AC which included an IGF profile then proceeded into an IVF \pm ICSI treatment cycle within 3-months. The first column shows the clinical outcomes among those women who utilized rGH daily as an adjuvant $(n=98)$ because they were considered to be poorprognosis cases, and from which group the 90 women represented in Table 5 were drawn. The second column shows the clinical outcomes for those women who were mostly considered to have a normal prognosis and did not utilize rGH adjuvant $(n=843)$.

\begin{tabular}{|l|l|l|}
\hline & $98 \mathrm{GH}+$ & $843 \mathrm{GH}-$ \\
\hline ART cycles (IVF \& FETs; $n$ ) & 98 & 843 \\
\hline ART cycles/ woman & 3.4 & 2.4 \\
\hline Age range at cycle (years) & $28-51$ & $25-47$ \\
\hline Oocyte/OPU ( $n$ ) & 6.1 & 11.1 \\
\hline Oocyte utilization rate & $25.0 \%$ & $28.7 \%$ \\
\hline Embryo utilization rate & $59.3 \%$ & $59.4 \%$ \\
\hline Pregnancy productivity rate/ Initiated cycle & $24.5 \%$ & $64.5 \%$ \\
\hline Pregnancy productivity rate / OPU & $27.9 \%$ & $71.0 \%$ \\
\hline Pregnancy productivity rate / ET & $38.1 \%$ & $85.3 \%$ \\
\hline Miscarriage \pm ectopic rate* & $6.1 \%$ & $6.9 \%$ \\
\hline Livebirth productivity rate / Initiated cycle & $18.4 \%$ & $49.6 \%$ \\
\hline Livebirth productivity rate / OPU & $20.9 \%$ & $54.6 \%$ \\
\hline Livebirth productivity rate / ET & $28.6 \%$ & $65.5 \%$ \\
\hline
\end{tabular}

IVF: in vitro fertilization; FET: frozen embryo transfer; OPU: oocyte pick-up; ET: embryo transfer *miscarriage: defined as those pregnancies reaching ultrasound definition (7 weeks)

* Ectopic: defined as pregnancy with laparoscopic retrieval of products of conception; pregnancies of unknown location classified as "biochemical” therefore not included.

\section{Discussion}

With the improved prognosis for IVF in recent years, we have been keen to optimise management in ART by defining good-prognosis versus poor-prognosis whilst the woman is still IVF-naïve. In this respect our AC enables the delineation of those cases which might benefit from adjuvant therapy prior to embarking on the complex and expensive IVF process. 
At present, based on our earlier studies $[2,4,5]$ we currently predict poor-prognosis on the basis of advanced female age (>40 years) and/or extremely low ovarian reserve based on AFC numbers and AMH levels. At PIVET this group constitutes $10 \%$ of new cases presenting for ART, however this rate is expected to rise as the proportion of older women seeking fertility treatment, rises. It is this group on whom we have focussed studies using rGH as an adjuvant.

In keeping with known physiology of hGH release from the pituitary into the vascular circuit [24,25,26] it is understood that hGH levels may be low in morning blood samples, even if the patient does not have AGHD. Our recent study measuring hGH levels on morning samples during ACs, showed that only $12 \%$ of women will have levels $>3.0 \mathrm{ng} / \mathrm{mL}$, thus excluding the need for further investigation for AGHD. However, in the same population, shown in Table 6), we have determined that $90 \%$ of those very same women have a good-prognosis in ART. The determination of AGHD from those women with low hGH levels would require a provocative challenge test, such as the insulin tolerance test (ITT). Although the ITT is regarded as the gold standard, in Australia the Endocrine Society and Medicare require the test to be performed by an Endocrinologist with the patient in a hospital setting, able to be resuscitated if profound hypoglycaemia occurs. Therefore, tests of lower risk involving glucagon stimulation (which induces insulin release) or clonidine challenge (binds to $\alpha 2$ adrenergic receptors) or a GH releasing hormone-arginine test (which inhibits somatostatin) may suffice [9]. Whichever provocative test is performed, they are all conducted over 3-4 hours with hourly blood sampling, seeking any reading of hGH $>3.0 \mathrm{ng} / \mathrm{mL}$ to exclude the diagnosis of AGHD.

Because of the complexity and limitations surrounding hGH testing, the simpler expediency of measuring IGF-1 is popular for those working with children and may suffice for adults. This has been accepted by Endocrine societies in the United States, the United Kingdom and Australia in recent years [9,11]. The IGF ratio which better reflects the proportion of IGF-1 which is unbound (from mainly IGFBP-3) in the serum, and presumably available for binding onto IGF receptors in tissues, is considered a better appraisal. In children IGF-1 levels can be matched with sub-optimal growth rates or deficiency in stature, to complete the diagnosis. In adults, such as those presenting for ART in this study, stature is unrelated [12], hence cases with low IGF-1 will require hGH testing followed by a provocative challenge test such as the ITT if levels are $<3.0 \mathrm{ng} / \mathrm{mL}$. With respect to IGF ratio, the normal range is considered between 1.6 to 5.0 ; ratios $<1.6$ matching excess (acromegaly, gigantism) and levels $>5.0$ matching GHD for either children or adults.

Given the relevance of applying the IGF profile, this study has explored whether rGH given as an adjuvant in the lead-up phase to an IVF treatment cycle, can change the profile in a positive way. Indeed, the data presented shows that the IGF1 levels increase significantly after 4-6 weeks of rGH given daily at a dose of just $1 \mathrm{IU}$, considered to match the normal physiological dose in adults $(24,25)$. With respect to the ovary it is known that the interaction of hGH with its receptor GHR can initiate canonical and non-canonical downstream signaling [26]. In canonical signaling, hGH from the pituitary stimulates liver cells to release IGF-1 (as well as IGF-2) into the circulation via transcription factors. In non-canonical signaling, the release of IGF-1 is independent of transcription factors but it does involve GH-GHR intracellular signaling leading to the recruitment of cytoplasmic enzymes including tyrosine kinases, phospholipases and protein kinases [26]. Whilst the specific mechanisms are not yet fully elucidated, it is clear that the GH-IGF axis is a key growth factor system involved in folliculogenesis [27, 28]. In vertebrates, the systemic IGF system comprises IGF-1, IGF-2, type-1 IGF receptor $(\mathrm{IGF}-1 \mathrm{R}), \mathrm{IGF}_{-2} \mathrm{R}$ and six IGF binding proteins that regulate IGF bioavailability; of which IGFBP-3 appears the most relevant in humans. The effect of IGFBP-3 is to increase the half-life of IGF-1 in the circulation and block its binding to the insulin receptor. In addition, it has been shown to modulate IGF-1 signaling to provide targeted adjustment and finetuning under various conditions [29]. As with IGF-1, the bulk of IGFBP-3 is also produced in the liver, although a wide range of other tissues produce smaller amounts. It is worth noting that hepatocytes are highly specialized cells which do not express IGF or IGFBP receptors thus enabling optimal secretion of these proteins into the circulation. Both IGF1 and IGFBP-3 are generated under the influence of hGH and this explains why our study showed significant elevations of both proteins under the influence of the adjuvant $\mathrm{rGH}$ treatment. Whilst there remain many unanswered questions about the wider range of functions for IGFBPs, the modulating influence of IGFBP-3 on IGF-1 is seen to be very important in the interplay of GH and IGF effects within reproductive tissues [26,29].

Because of the modifying influence of IGFBPs on IGF-1 function, it has been proposed that the ratio of the two proteins provides a better measure of IGF-1 function. One Danish study applied a ratio of IGF-1/IGFBP-3, demonstrating a decline in otherwise healthy, aging adults and a significant elevation in acromegalic patients [30]. It should be noted that inversion of the ratio (as with our studies, to avoid fractional expression) interprets a reverse observation indicating that a high IGFBP-3/ IGF-1 ratio reflects GHD and a low ratio reflects acromegaly. Similarly, a recent study from Tunisia in children indicates that the IGF ratio provides a clearer relationship with the clinical features for utilizing growth hormone therapy in small-for-gestational-age (SGA), growth hormone-deficient (GHD) and Prader-Willi (PWS) children [31]. Again, the study reports IGF-1/ IGFBP-3 ratios as fractional showing rises following rGH therapy (from $0.14 \pm 0.04$ to $0.19 \pm 0.07$ in SGA cases; from $0.07 \pm 0.01$ to $0.20 \pm 0.08$ for the GHD group; and from $0.12 \pm 0.02$ to $0.19 \pm 0.09$ for the PWS group). Our preference for the inverted ratio of IGFBP-3/IGF-1 would show a declining ratio in a clearer manner. 
However, we agree that the IGF ratio, in principle, provides a clearer picture for assessing clinical GHD. In our study, both IGF-1 and IGFBP-3 both showed significant elevations under the influence of rGH adjuvant treatment.

With respect to clinical outcomes in ART related to IGF-1 levels and IGF ratios, there has been little so far reported. In the search for biomarkers which can predict IVF cycle cancellation due to poor ovarian responses, a group from New York [32] showed significant relevance for reduced AFCs and AMH levels as predictive and, surprisingly from our perspective, elevated IGF-1 levels along with soluble fms-like tyrosine kinase (sFLT-1) significantly associated with poor response and cancelled IVF cycles. More recently, a different study from the same institute in New York reported on IGF-1 as a predictive tool for ovarian hyperstimulation outcomes in poor-responders [33]. Again, surprisingly from our perspective, the study showed that Day-2 IGF-1 levels were higher in those women who had a poor response and negative outcomes in their IVF treatment cycle. Luteal phase pre-treatment, as yet another IVF adjuvant was associated with improved clinical outcomes and this was associated with, again surprising to us, a reduction in IGF-1 levels. These recent IVF-related studies [32,33] indicate that the notion of poor-prognosis may not be an AGHD disorder, hence wellstructured research projects will be required to clarify this.

\section{Conclusion}

In this fourth study examining the relevance of measuring the IGF profile (IGF-1, IGFBP-3 and the ratio of IGFBP-3/ IGF1) in women presenting for assisted reproductive technologies (ART), we have examined the influence of recombinant growth hormone $(\mathrm{rGH})$ prescribed as an adjuvant on the profile of IVF-naïve women predicted to have a poor-prognosis on the basis of advanced age $>40$ years or very low AFCs and/or AMH levels. The data showed that rGH caused a significant elevation in IGF-1 as well as its main binding protein, IGFBP-3, albeit to a lesser degree. Consequently, the IGF ratio, which we measure as IGFBP-3/ IGF-1 and which is considered a more reliable marker of IGF-1 bioactivity, was also shown to be significantly reduced towards the normal range. The data of clinical outcomes from the 90 women in the study group as well as the entire 941 women who had IGF profiles undertaken in a preliminary AC before entering the IVF \pm ICSI treatment are presented for comparison. We have noted these clinical data are not suitable for statistical evaluation as the groupings are disparate and unmatched. None-the-less, categorization clearly expressed the poorprognosis groupings were correct and shows a difference in clinical outcomes with the poor-prognosis group achieving livebirths at $21 \%$ of OPU cycles, whilst the remainder had higher rates at $55 \%$ across the broad age range. Further studies are intended to show the clinical relevance of a higher IGF-1 level for clinical outcomes from IVF as well as the relevance of increasing the IGF-1 level by using rGH as an adjuvant.

\section{Compliance with ethical standards}

\section{Acknowledgments}

We are grateful for the close working relationship between PIVET $®$ Medical Centre and CLINIPATH $®$ Pathology which carried out the assays involved in the IGF profile and is also accredited by NATA.

\section{Disclosure of conflict of interest}

The entire project has been funded internally at PIVET without any external or commercial contributions. The authors declare no conflict of interest.

\section{Statement of ethical approval}

Reporting of this data was approved under Curtin University Human Ethics Committee approval no. RD_25-10 general approval for retrospective data analysis in 2010, updated in 2015, and again further updated recently, in August 2020.

\section{Statement of informed consent}

PIVET is accredited with both the self-regulatory National Australian Reproductive Technology Committee (RTAC) as well as the Reproductive Technology Council (RTC) of Western Australia. Consent forms received approval under both regulatory bodies. The assay laboratory is accredited on an annual basis by the National Australian Testing Authority (NATA).

\section{Author Contributions}

The study was conceived by PIVET Medical Director JLY who established the data-base at PIVET Medical Centre with the assistance of IT Consultant and data manager PMH. The first data extractions were undertaken by SZ who was on a 
sabbatical study period from her facility in Malaysia to which she has now returned. The Data has been further analysed by PMH supported by MDKN who has a managerial role for the Laboratory Assays at PIVET. All authors have assisted with the data analyses as well as the preparation of the Tables and Figures. The manuscript was written by JLY and each of the authors have read and agreed to its content.

\section{References}

[1] Annual Capri Workshop Group. IVF, from the past to the future: the inheritance of the Capri Workshop Group. Hum Reprod Open, 2020; pp1-9, doi: 10.1093/hropen/hoaa040

[2] Yovich JL, Stanger JD. Growth hormone supplementation improves implantation and pregnancy productivity rates for poor-prognosis patients undertaking IVF, Reprod Biomed Online, 2010; 21: 37-49: doi: 10.1016/j.rbmo.2010.03.013

[3] Keane KN, Yovich JL, Hamidi A, Hinchliffe PM, Dhaliwal SS. A single-centre retrospective analysis of growth hormone supplementation in IVF patients classified as poor-prognosis. BMJ Open, 2017; 7(e018107):1-9, doi: doi:10.1136/bmjopen-2017-018107

[4] Keane KN, Ye Y, regan SLP, Dhaliwal SS, yovich JL. Live birth outcomes of vitrified embryos generated under growth hormone stimulation are improved for women categorized as poor-prognosis. Clin Exp Reprod Med, 2019; 46(40):178-188, doi: 10.5653/cerm.2019.00206

[5] Yovich JL, Ye Y, Regan SLP, Keane KN. The evolving concept of poor-prognosis for women undertaking IVF and the notion of growth hormone as an adjuvant; a single-center viewpoint. Front Endocrinol, 2019; 10:808; pp14, doi.org/10.3389/fendo.2019.0080

[6] Yovich JL, Regan SL, Zaidi SN, Keane KN. The concept of growth hormone deficiency affecting clinical prognosis in IVF. Front Endocrinol, 2019; 10: 650, doi: 10.3389/fendo.2019.00650

[7] Weall BM, Al-Samerria S, Conceicao J, Yovich JL, Almahbobi G. A direct action for GH in improvement of oocyte quality in poor-responder patients. Reproduction, 2015; 149(2):147-154, doi: 10.1530/REP-14-0494

[8] Regan SLP, Knight PG, Yovich JL, Arfuso F, Dharmarajan A. Growth hormone during in vitro fertilization in older women modulates the density of receptors in granulosa cells, with improved pregnancy outcomes. Fertil Steril 2018; 110: 1298-1310.

[9] Yovich JL, Zaidi S, Nguyen MDK, Hinchliffe PM. Measuring IGF-1 and IGFBP-3 profiles in women seeking assisted reproduction; relationship to serum growth hormone levels (Study 3). GSC Biological and Pharmaceutical Sciences, 2020; 13(03), 032-053.

[10] Stanley T. Diagnosis of growth hormone deficiency in childhood. Curr Opin Endocrinol Diabetes Obes, 2012; 19: 47-52, doi: 10.1097/MED. 0b013e32834ec952

[11] Molitch ME, Clemmons DR, Malozowski S, Merriam GR, Vance ML. Evaluation and treatment of adult growth hormone deficiency: An Endocrine Society Clinical Practice Guideline. J Clin Endocrinol Metab, 2011; 96(6):1587-1609, doi: 10.1210/jc.2011-0179

[12] Yovich JL, Zaidi S, Nguyen MDK, Hinchliffe PM. Measuring IGF-1 and IGFBP-3 profiles in women seeking assisted reproduction; relationship to clinical parameters (Study 1). J Pers Med,2020; 10:122 doi: 10.3390/jpm10030122

[13] Yovich JL, Zaidi S, Nguyen MDK, Hinchliffe PM. Measuring IGF-1 and IGFBP-3 profiles in women seeking assisted reproduction; relationship to ovarian reserve parameters (Study 2). GSC Biological and Pharmaceutical Sciences, $2020 ; 13(02), 035-053$.

[14] Yovich J, Stanger J, Hinchliffe P. Targeted gonadotrophin stimulation using the PIVET algorithm markedly reduces the risk of OHSS. Reprod Biomed Online, 2012; 24(3):281-292, doi: 10.1016/j.rbmo.2011.11.014

[15] Yovich JL, Alsbjerg B, Conceicao JL, Hinchliffe PM, Keane KN. PIVET rFSH dosing algorithms for individualized controlled ovarian stimulation enables optimized pregnancy productivity rates and avoidance of ovarian hyperstimulation syndrome. Drug Des Devel Ther, 2016; 10: 2561-573, doi: 10.2147/DDDT.S104104

[16] Yovich JL. How to Prepare the Egg and Embryo to Maximise IVF Success. In: Monitoring the stimulated IVF cycle. Section II: Stimulation for IVF (Eds: Gabor T Kovacs, Anthony J Rutherford, David K Gardner). Cambridge University Press, Cambridge, UK, 2019; 94-120 doi: 10.1017/9781316756744 
[17] Yovich JL, Hinchliffe PM, Lingam S, Srinivasan S, Keane KN. Adjusting the PIVET rFSH dosing algorithm for the biosimilar Bemfola product. J Fertil In vitro IVF Worldw Reprod Med Genet Stem Cell Biol, 2018; 5:3, doi: 10.4172/2375-4508.1000207

[18] Yovich JL, Craft IL. Founding pioneers of IVF: Independent innovative researchers generating livebirths within 4 years of the first birth. Reprod Biol, 2018; 18:317-323, doi.org/10.1016/J.repbio.2018.11.004

[19] Yovich JL. Founding pioneers of IVF Update: Independent innovative researchers generating livebirths within 4 years of the first birth. Reprod Biol, 2020; 20:111-113, doi.org/10.1016/j.repbio.2019.12.008

[20] Kuwayama M, Vajta G, Kato O, Leibo SP. Highly efficient vitrification method for cryopreservation of human oocytes. Reprod Biomed Online, 2005; 11:300-308, doi: doi: 10.1016/s1472-6483(10)60837-1

[21] Newman JE, Paul RC, Chambers GM. Assisted reproductive technology in Australia and New Zealand 2018. Sydney: National Perinatal Epidemiology and Statistics Unit, the University of New South Wales, Sydney, 2020; $1-83$.

[22] Hammer $\emptyset$, Harper DAT, Ryan PD. PAST: Paleontological Statistics software package for education and data analysis. Palaentologia Electronica 2001; 4(1): 9pp

[23] Norman RJ, Alvino H, Hull LM, Mol BW, Hart RJ, Kelly TL, Rombauts L. Human growth hormone for poor responders: a randomized placebo-controlled trial provides no evidence for improved live birth rate. Reprod Biomed Online, 2019; 38:908-915, doi: 10.1016/j.rbmo.2019.02.003

[24] Hull KL, Harvey S. Growth hormone and reproduction: a review of endocrine and autocrine, paracrine interactions. Int J Endocrinol, 2014; Article ID 234014: pp24, doi: 10.1155/2014/234014

[25] Steyn FJ, Tolle V, Chen C, Epelbaum J. Neuroendocrine regulation of growth hormone secretion. Compr Physiol, 2016; 6(2):687-735, doi: 10.1002/cphy.c150002. PMID: 27065166.

[26] Ipsa E, Cruzat VF, Kagize JN, Yovich JL, Keane KN. Growth Hormone and Insulin-like growth factor in reproductive tissues. Front Endocrinol 2019; 777 pp14 doi: 10.3389/fendo.2019.00777

[27] Matsuda F, Inouie N, Manabe N, Okhura S. Follicular growth and atresia in mammalian ovaries: regulation by survival and death of granulosa cells. J Reprod Dev, 2012; 58:44-50, doi: 10.1262/jrd.2011-012

[28] Devesa J, Caicedo D. The role of growth hormone on ovarian functioning and ovarian angiogenesis. Front Endocrinol, 2019; 10:pp16, doi: 10.3389/fendo.2019.00450

[29] Allard JB, Cunming D. IGF-binding proteins: why do they exist and why are there so many? Front Endocrinol, 2018; 9:117, pp12, doi: 10.3389/fendo.2018.0017

[30] Juul A, Main K, Blum WF, LindholmJ, Ranke MB, Skakkebaek NE. The ratio between serum levels of insulin-like growth factor (IGF)-1 and the IGF binding proteins (IGFBP-1,2, and 3) decreases with age in healthy adults and is increased in acromegalic patients. Clin endocrinol (oxf), 1994; 41(1):85-93, doi: 10.1111/j.13652265.1994.tb03788.x

[31] Gaddas M, Périn L, Le Bouc Y. Evaluation of IGF1/IGFBP3 molar ratio as an effective tool for assessing the safety of growth hormone therapy in small-for-gestational-age, growth hormone-deficient and Prader-Willi children. J Clin Res Pediatr Endocrinol 2019; 11(3): 253-261, doi: 10.4274/jcrpe.galenos.2019.2018.0277

[32] Nasioudis D, Minis E, Irani M, Kreines F, Witkin SS, Spandorfer SD. Insulin-like growth factor-1 and soluble FMSlike tyrosine kinase-1 prospectively predict cancelled IVF cycles. J Assist Reprod Genet, 2019; 36(12):2485-2491, doi: 10.1007/s10815-019-01618-3.

[33] Man L, Lekovich J, Canon C, Rosenwaks Z, James D. 2019. Cycle day-2 insulin-like growth factor-1 serum levels as a prognostic tool to predict controlled ovarian hyperstimulation outcomes in poor responders. Fertil Steril 2020; 113: 1205-1214. Doi.org/10.1016/j.fertnstert.2020.02.111 